\title{
MODIFIED MODELS FOR LOVE AND THEIR DYNAMICAL PROPERTIES
}

\author{
KEWANG CHEN, WENJUN LIU, AND JAEKEUN PARK
}

Received 06 October, 2015

\begin{abstract}
In this work, we modify the present linear and nonlinear dynamic models of love between two individuals. For the linear system, we make the model more realistic by introducing external perturbation functions, and then discuss the influences of different types of external perturbation functions. For the nonlinear system, we focus on the love affairs between two "complementary people" and introduce the nonlinearities in a different way. Then we investigate the corresponding mathematical properties of the systems trajectories and phase-plane, and explain its special meanings in a romantic relationship. Also, we have offered some practical advice to promote the development of their relationship.
\end{abstract}

2010 Mathematics Subject Classification: 91D10; 93A30; 37D45

Keywords: love dynamic, differential equations, external perturbation function, singular point, limit cycle

\section{INTRODUCTION}

Mathematics is powerful, but this power has rarely been applied to the dynamics of romance. Rapoport [9] and Radzicki [8] firstly introduced a simple mathematical model to discuss love affairs. Strogatz [16] gave a short discussion on Rapoport's models and presented several related mathematic exercises. By taking into account the appeal that each partner presents to the others in absent of other feelings, Rinaldi [10] proposed a modified version of of the Strogatz's linear model. Gottman [5] presented dynamical models of the verbal interaction of married couples. Sprott [14] proposed a sequence of dynamical models involving coupled ordinary differential equations describing the time variation of the love or hate displayed by individuals in a romantic relationship. Sportt started with the analysis of a linear system of two individuals. Then he went further to study love triangles, and finally made the inclusion of the effect of nonlinearities and demonstrated the origin of chaos. For other related results and interesting applications, the reader may refer to $[1,2,4,7$, $11-13,17,19,20]$ and the references therein.

In any models of love, it is obviously difficult to define what is love and how to quantify it in some meaningful way [3]. There are many different kind of love, 
such as passion, intimacy, and commitment [15], and each type consists of a complex mixture of feelings. Besides, there is love of oneself, love of life, and so forth. What's more, the opposite of love may not be hate, since the two feelings can coexist, and one can love something about one's partner and hate others at the same time. Therefore, the models mentioned above can be made more realistic by introducing additional phenomena and parameters.

In this paper, we modify both linear and nonlinear models of love between two individuals. For the linear system, we introduce the external influences or perturbation function $f(t)$ in Section 2. By considering different types of $f(t)$, we will show that the dynamics of the system is rich and interesting. For nonlinear system, we introduce nonlinearities in a different way and focus on two complementary lovers in Section 3. Based on the new model we construct, we investigate the properties of its trajectory and phase plane and explain its special meanings in love affairs. We also give some practical suggestions to promote the development of their love.

\section{Linear System with Perturbation FunCtion}

In [16], Strogatz considered a love affair between Romeo and Juliet, where $R(t)$ is Romeo's love for Juliet at time $t$ and $J(t)$ is Juliet's love for Romeo at the same instant. The standard Strogatz linear model is given by

$$
\begin{aligned}
& \frac{\mathrm{d} R}{\mathrm{~d} t}=a R+b J, \\
& \frac{\mathrm{d} J}{\mathrm{~d} t}=c R+d J,
\end{aligned}
$$

where $a$ and $b$ specify Romeo's romantic style, and $c$ and $d$ specify Juliet's style. The parameter $a$ describes the extent to which Romeo is encouraged by his own feelings, and $b$ is the extent to which he is encouraged by Juliet's feelings. The parameters $d$ and $c$ have the equivalent significance from the perspective of Juliet. These four parameters can be positive or negative, Romeo can exhibit of four romantic styles depending on the signs of $a$ and $b$, which are given below:

(1) Eager beaver: $a>0, b>0$ (Romeo is encouraged by his own feelings as well as Juliet's)

(2) Narcissistic nerd: $a>0, b<0$ (Romeo wants more of what he feels but retreats from Juliet's feelings)

(3) Cautious (or secure) lover: $a<0, b>0$ (Romeo retreats from his own feelings but is encouraged by Juliet's)

(4) Hermit: $a<0, b<0$ (Romeo retreats from his own feelings as well as Juliet's) The same format can be generated from Juliet involving the parameters $c$ and $d$.

A modified version of the Strogatz's linear model was proposed by Rinali [10] in which a constant term was added to each of the derivatives in the system of equations of (2.1) to take into account the appeal or repulsion that each partner presents to the 
other in absence of other feelings. Then the model can be written as follows:

$$
\begin{aligned}
& \frac{\mathrm{d} R}{\mathrm{~d} t}=a R+b J+p, \\
& \frac{\mathrm{d} J}{\mathrm{~d} t}=c R+d J+q .
\end{aligned}
$$

This model is more realistic since it allows feelings to grow from a state of indifference and it provides an equilibrium not characterized by complete apathy [14]. By calculating, we can obtain that the equilibrium point for system $(2.1)$ is $(0,0)$ and for system (2.2) is $\left(\frac{b q-d p}{a d-b c}, \frac{a q-c p}{a d-b c}\right)$ (assuming $a d \neq b c$ ).

Now, since it is obviously unrealistic to exclude other external influences or perturbation (such as Juliet's losing her job, or Romeo has a negative expectation for their future, or other perturbations which may have an effect on their love for each other), we want make the model more reasonable. Here, we introduce an external influence or perturbation function $f(t)$ for Romeo and $g(t)$ for Juliet, and then the model can be written as follows:

$$
\begin{aligned}
& \frac{\mathrm{d} R}{\mathrm{~d} t}=a R+b J+f(t), \\
& \frac{\mathrm{d} J}{\mathrm{~d} t}=c R+d J+g(t) .
\end{aligned}
$$

From the new model we construct, we notice that if we neglect the external influence i.e. $f(t)=0, g(t)=0$, then we get system (2.1) and if we set $f(t)=p, g(t)=q$, where $p$ and $q$ are constant, then we get system (2.2). This means the new perturbation function includes the previous internal influence. By considering different types of perturbation function, we obtain some interesting results, as shown below.

Typically, Gragnani, Rinaldi, and Feichtinger [6] pointed out that the majority of the people is secure lovers. Here, we suppose Romeo representing the majority of people, which means he is a cautious lover with $a$ being negative and $b$ positive and assume Juliet is Narcissistic nerd with negative $c$ and positive $d$. We will show how their love dynamics develop in different situations with corresponding various types of perturbation function $f(t)$ and $g(t)$.

To begin with, we exclude the external perturbation terms in the system, then the result is shown in Figure 1 (here $a=-1, b=2, c=-2, d=1, f(t)=0, g(t)=0$, initial value is $(0.1,0.1))$. One has difficult to foretell the ending of their love story, everything is possible. This result is logically accepted!

Then we go further, supposing that Juliet gets a surprise from Romeo such as receiving an unexpected gift or other kind of romantic thing. This situation can be described by letting $g(t)$ be a stimulus function, which only have value in some point (be it the day when Juliet gets a surprise from Romeo !). 

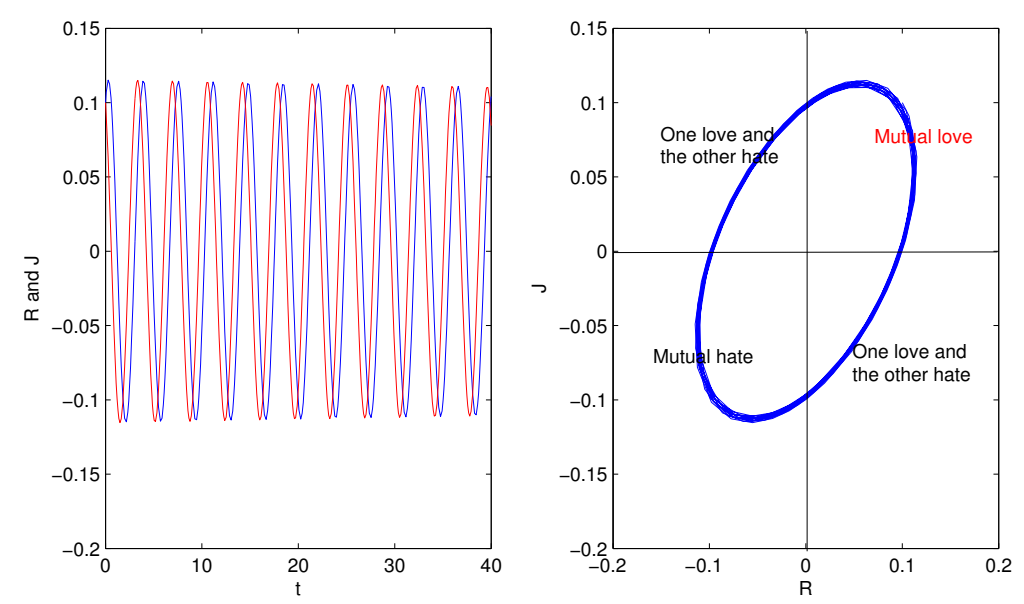

FIGURE 1. Trajectory of system (2.3), where $a=-1, b=2, c=$ $-2, d=1, f(t)=0, g(t)=0$.

For example (see also Figure 2)

$$
g(t)= \begin{cases}12, & \text { if } t=10 \\ 0, & \text { otherwise }\end{cases}
$$

This situation and results are shown in Figure 2 (here $a=-1, b=2, c=-2, d=1$, $f(t)=0$, initial value is $(0,0), g(t)$ is a stimulus function, given by $(2.4))$. Then the phase plane suggests that this kind of perturbation or romantic stimulus may result in mutual love. This is quite true in common sense!

Moreover, we assume that Romeo gets a new satisfied job and be confident in their future life. In this kind of situation, the result is shown in Figure 3 (here $a=-1$, $b=2, c=-2, d=1, f(t)=0.03 t, g(t)=0$, initial value is $(0.1,0.1))$. Here we consider the time interval within a month (i.e., 30 days), so the value of the forcing function will not go infinite within a month. One can figure out from the Figure 3 that their relationship is developing towards mutual love!

Finally, we consider a small-periodic perturbation situation, which represents the influence of all kinds of daily trifles. The outcome is shown in Figure 4 (here $a=-1$, $b=2, c=-2, d=1, f(t)=\sin (0.2 \pi t), g(t)=0$, initial value is $(0.1,0.1))$. In stimulation, the amplitude values are chosen corresponding to the value of $R$ and $J$, which is neither too small nor too big. The resulting dynamics are quasi-periodic and produce a periodic cycle. One can even harder to foretell the development of their relationship. 


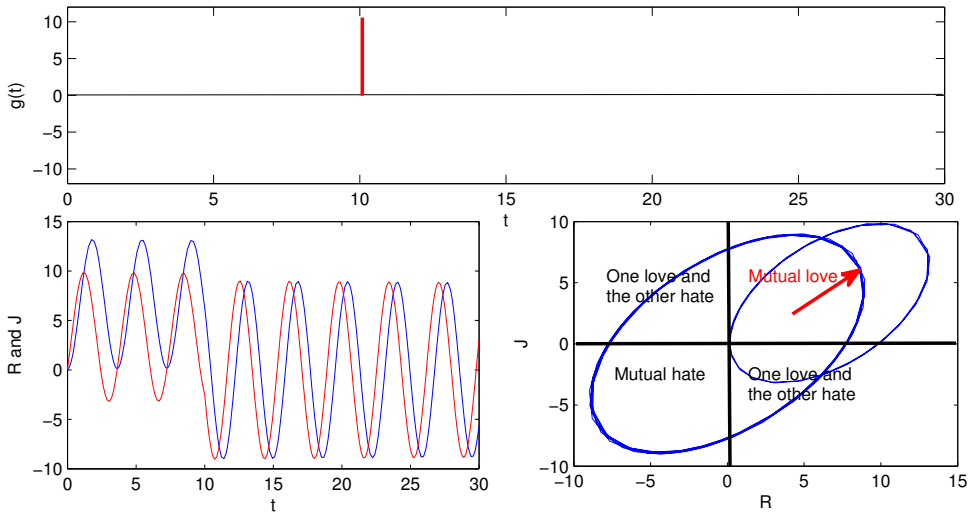

FIGURE 2. Trajectory of system (2.3), where $a=-1, b=2, c=$ $-2, d=1, f(t)=0, g(t)$ is a stimulus function.
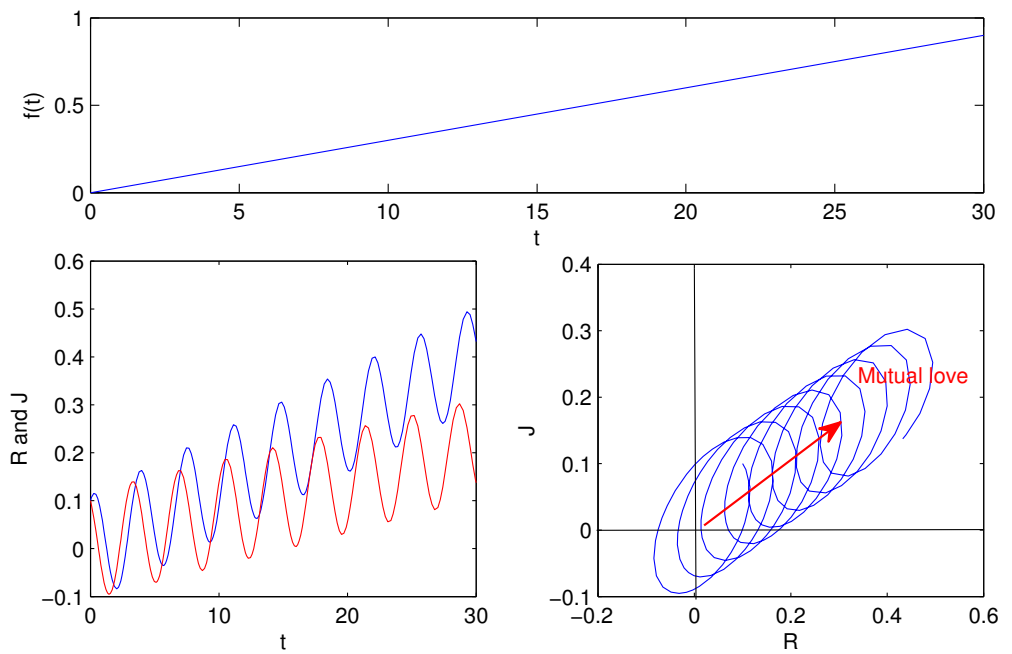

FIGURE 3. Trajectory of system (2.3), where $a=-1, b=2, c=$ $-2, d=1, f(t)=0.03 t, g(t)=0$.

\section{NONLINEAR SYSTEM FOR COMPLEMENTARY COUPLES}

The forgoing discussion involved only linear equations for which the allowable dynamics are limited. There are countless ways to introduce nonlinearities. Here 

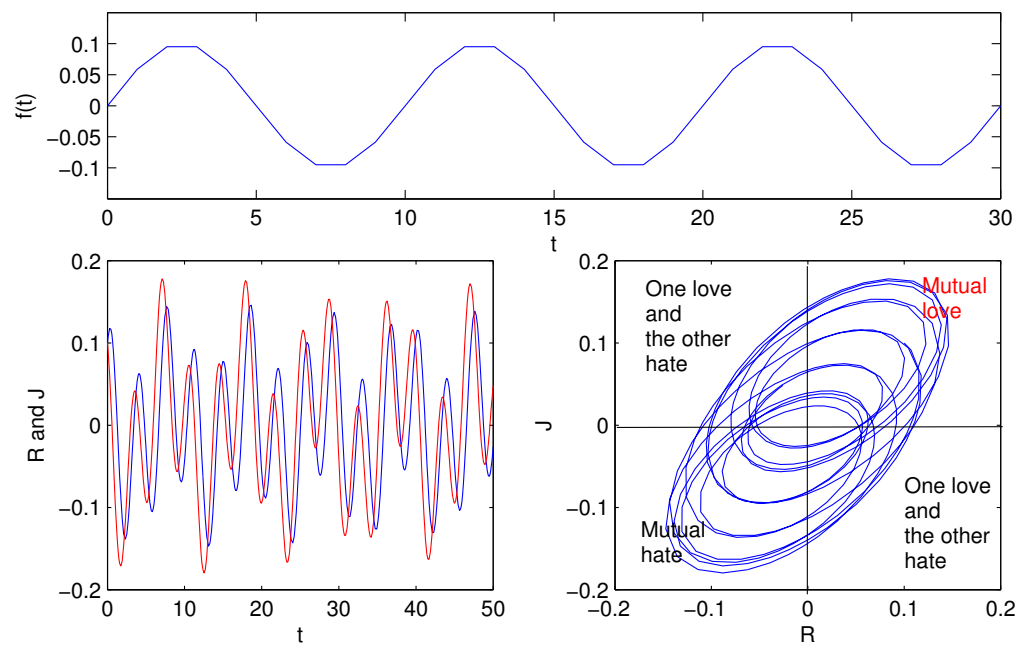

FIGURE 4. Trajectory of system (2.3), where $a=-1, b=2, c=$ $-2, d=1, f(t)=\sin (0.2 \pi t), g(t)=0$.

we study the love affairs between complementary people and introduce nonlinearity in a different way. The "complementary people" here means people who have complementary personality characteristics such as one is rational while the other is emotional. Now, we suppose that Juliet responds positively to Romeo's love. But if Romeo loves her too much beyond a level of comfort, she starts to react adversely. Conversely, if Romeo is adequately hostile, Juliet may decide to be nice to him. Gottman et al. [5] termed it as "repair nonlinearity". Sprott [14] formulated the above phenomenon by replacing $c R$ in system (2.1) with logistic function $c R(1-R)$. This states that $R=1$ corresponds to the value at which Romeo's love becomes counterproductive. But in reality this is violating the variability in human nature. The levels to which should be different from people to people. For example, for Romeo, we use $R_{0}$ represents the certain level that Juliet may react adversely when $R>R_{0}$; for Juliet, we may use $J_{0}$ represents the certain level that Romeo react adversely when $J>J_{0}$. So we use $c R\left(1-\frac{R}{R_{0}}\right)$ instead of $c R(1-R)$. Different to Juliet, Romeo may behave quite differently since they have complementary personality characteristics. Here, we suppose Romeo is a kind of person who is always wanting more love from the other and he will not be smothered by the love of his partner. Moreover, from the past there are no restrictions on the boundary of one's love. Here we suppose Romeo is a rational person and his totally love can be bounded by $E$ ( $E$ represents the maximum value of Romeo's love $R$, and $E>R_{0}$ ), which means his love for Juliet can not go infinite. Then, it is reasonable to say that the change of Romeo's love 
for Juliet $\frac{\mathrm{d} R}{\mathrm{~d} t}$ is proportional to the present value $R$ and its potential ability $\frac{E-R}{E}$, i.e., replacing $a R$ in system (2.1) with $a R\left(\frac{E-R}{R}\right)$. Although we can suppose Juliet's love is bounded too, this will make the model too complex, so here we will not go that far to limit her love. Another reason for not limiting Juliet's love is that Juliet and Romeo are complementary people, so Juliet might be more emotional, which means her love can be unbounded. If we also include the external influence or perturbation in the system, then the general nonlinear system for complementary couples reads as follows:

$$
\begin{aligned}
& \frac{\mathrm{d} R}{\mathrm{~d} t}=a R\left(\frac{E-R}{E}\right)+b J+f(t), \\
& \frac{\mathrm{d} J}{\mathrm{~d} t}=d J+c R\left(1-\frac{R}{R_{0}}\right)+g(t) .
\end{aligned}
$$

In order to investigate some mathematics properties, here we just consider certain kind of people's love affairs and exclude the external influence, i.e., $f(t)=0$ and $g(t)=0$. Similar to the linear system situation, we suppose Juliet is a secure lover $(d<0, c>0$, here we call $d$ as a "caution coefficient") and Romeo is a Narcissistic nerd ( $a>0, b<0$, here we call $a$ as a "narcissism coefficient"). If we suppose all the coefficients $\left(a_{0}, b_{0}, c_{0}, d_{0}\right)$ are positive in the following system, then we get:

$$
\begin{aligned}
& \frac{\mathrm{d} R}{\mathrm{~d} t}=a_{0} R\left(\frac{E-R}{E}\right)-b_{0} J, \\
& \frac{\mathrm{d} J}{\mathrm{~d} t}=-d_{0} J+c_{0} R\left(1-\frac{R}{R_{0}}\right) .
\end{aligned}
$$

Set $u(t)=\frac{R(t)}{E}, v(t)=\frac{J(t)}{E}, b_{0} t=\tau$, then we have:

$$
\begin{aligned}
& \frac{\mathrm{d} u}{\mathrm{~d} \tau}=\frac{a_{0}}{b_{0}} u(1-u)-v, \\
& \frac{\mathrm{d} v}{\mathrm{~d} \tau}=-\frac{d_{0}}{b_{0}} v+\frac{c_{0} E}{b_{0} R_{0}}\left(\frac{R_{0}}{E}-u\right) u .
\end{aligned}
$$

We know that the system (3.2) and (3.3) are topological homeomorphism. If we assume $\frac{\left(a_{0}+d_{0}\right)^{2}}{4 b_{0} c_{0}}<1<\frac{E}{R_{0}}$ holds, then the trajectory of system (3.3) has the following properties, which has its special and interesting meanings in the romantic relationship:

Theorem 1. If $a_{0} \leq d_{0}$, system (3.3) has no closed path curve; system (3.3) has two finite singular points $(0,0)$ and $\left(u_{0}, v_{0}\right)$, where $\left(u_{0}, v_{0}\right)=\left(\frac{\left(a_{0} d_{0}-b_{0} c_{0}\right) R_{0}}{a_{0} d_{0} R_{0}-b_{0} c_{0} E}\right.$, $\left.\frac{\left(a_{0}-b_{0}\right)\left(a_{0} d_{0}-b_{0} c_{0}\right) R_{0}}{b_{0}\left(a_{0} d_{0} R_{0}-b_{0} c_{0} E\right)}\right) ;\left(u_{0}, v_{0}\right)$ is a saddle point in the first quadrant; $(0,0)$ is an stable strong focus when $a_{0}<d_{0}$. 
Theorem 2. For system (3.3), when $a_{0}=d_{0},(0,0)$ is a stable first-order weak focus; when $a_{0}>d_{0},(0,0)$ is an unstable strong focus; when $0<a_{0}-d_{0} \ll b_{0}$, there exists a unique limit cycle around point $(0,0)$, and limit cycle is single stable cycle; when $a_{0}-d_{0}>\frac{a_{0}\left(a_{0} d_{0}-b_{0} c_{0}\right) R_{0}}{b_{0}\left(a_{0} d_{0} R_{0}-b_{0} c_{0} E\right)}$, there are no limit cycles.

Remark 1. The detail proof of the above two theorems can be found in [18] and we shall give a brief one in Appendix for the convenience of readers. In the following, we just focus on its special meanings in love affairs.

Based on the Theorem 1 to Theorem 2 and the corresponding phase plane, we will explain its special meanings in the love affair between narcissistic Romeo and cautious Juliet. Also, practical advice is offered to improve their relationship.

Case (1): $a_{0}<d_{0}$. In this case, the caution coefficient of Juliet is larger than the narcissism coefficient of Romeo. According to Theorem 1, there is no closed path curves and there are two finite singular points $(0,0)$ and $\left(u_{0}, v_{0}\right)$. Here, $(0,0)$ is a strong stable focus and $\left(u_{0}, v_{0}\right)$ is a saddle point in first quadrant, as shown in Figure 5. We also notice that the two trajectories that approach the saddle point $\left(u_{0}, v_{0}\right)$, together with that saddle point, form a separatrix that separates Regions 1 and Regions 2 in Figure 5. It plays a crucial role in determining the long-term relationship between Romeo and Juliet. If the initial point $\left(R_{0}, J_{0}\right)$ lies precisely on the separatrix, that $(R(t), J(t))$ approach the saddle point $\left(u_{0}, v_{0}\right)$ as time goes by. Of course, random events make it extremely unlikely that $(R(t), J(t))$ will remain on the separatrix. If not, mutual love relationship is impossible theoretically. If the initial point $\left(R_{0}, J_{0}\right)$ lies in Region 1 above the separatrix, as time goes by, then $(R(t), J(t))$ will oscillates and spirals inward toward the origin, which means they may end up in mutual apathy. Alternatively, if the initial point $\left(R_{0}, J_{0}\right)$ lies in Region 1 below the separatrix, as time goes by, $J(t)$ will become negative although $R(t)$ may be positive, which means Romeo may addicted to Juliet while Juliet has the adverse feeling. This is because the caution coefficient of Juliet is larger than the narcissistic coefficient of Romeo.

Case (2): $a_{0}>d_{0}$ and $0<a_{0}-d_{0} \ll b_{0}$. In this case, the narcissism coefficient of Romeo is just a little larger than the the caution coefficient of Juliet. According to Theorem 2, there exists a limit cycle around the origin point $(0,0)$, as show in Figure 6 . The only difference is that when the initial point $\left(R_{0}, J_{0}\right)$ is in Region 1 , the $(R(t), J(t))$ will oscillates and spirals to a limit cycle around $(0,0)$. This means they won't end up in totally apathy, but they relationship oscillates around this state. Sometimes better, sometimes worse.

Case (3): $a_{0}>d_{0}$ and $a_{0}-d_{0}>\frac{a_{0}\left(a_{0} d_{0}-b_{0} c_{0}\right) R_{0}}{b_{0}\left(a_{0} d_{0} R_{0}-b_{0} c_{0} E\right)}$. In this case, the narcissism coefficient of Romeo is much larger than the the caution coefficient of Juliet. Then Theorem 2 implies that there exists no limit cycles, just as the phase plane shows in Figure 7. Except the two trajectories that can approaching the saddle point in Figure 


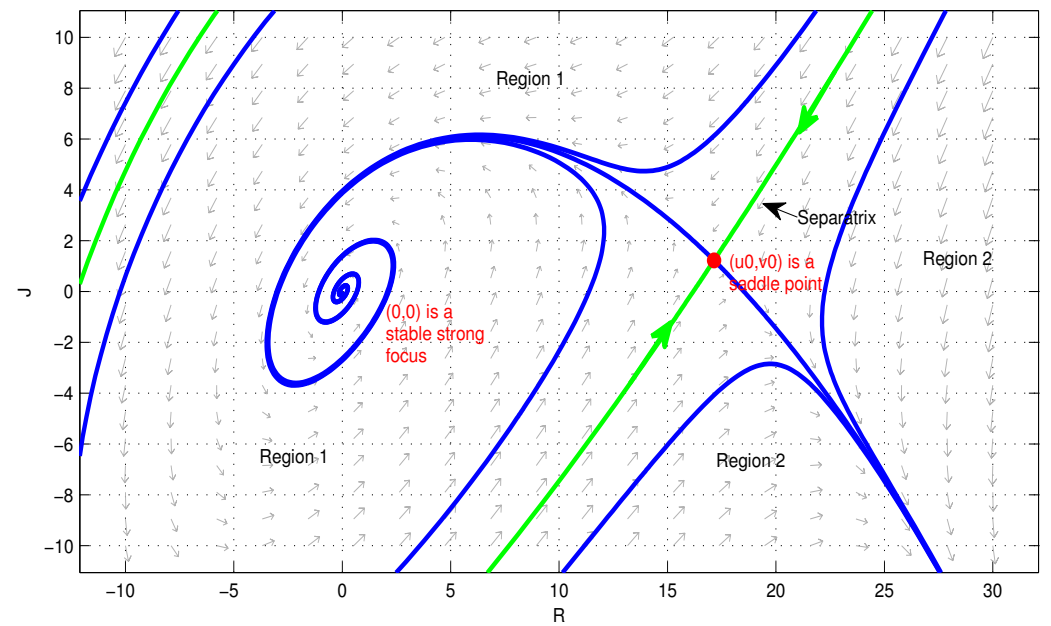

FIGURE 5. The phase plane of system (3.2) in the first case, where $a_{0}=0.5, b_{0}=1, c_{0}=1.2, d_{0}=0.8, E=20, R_{0}=18$.

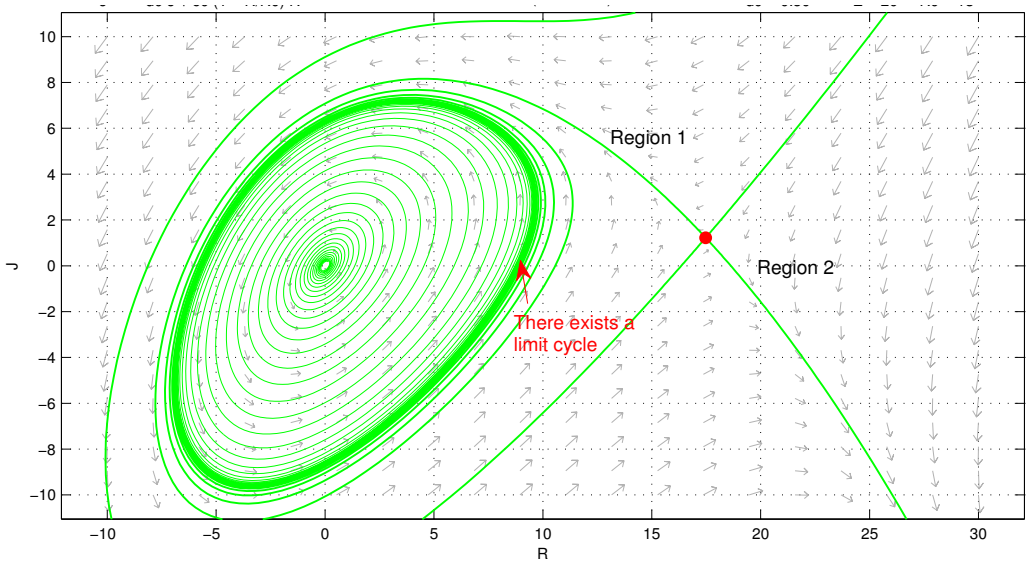

FIGURE 6. The phase plane of system (3.2) in the second case, where $a_{0}=0.555, b_{0}=1, c_{0}=1.2, d_{0}=0.5, E=20, R_{0}=18$.

7, it is nearly, theoretically, impossible for an over narcissistic person to fall in love with a cautious lover, no matter what the initial conditions are.

\section{Conclusions}

In the present work, we modify the linear love dynamic model for two individuals by introducing external perturbation function and show that the dynamics of this 


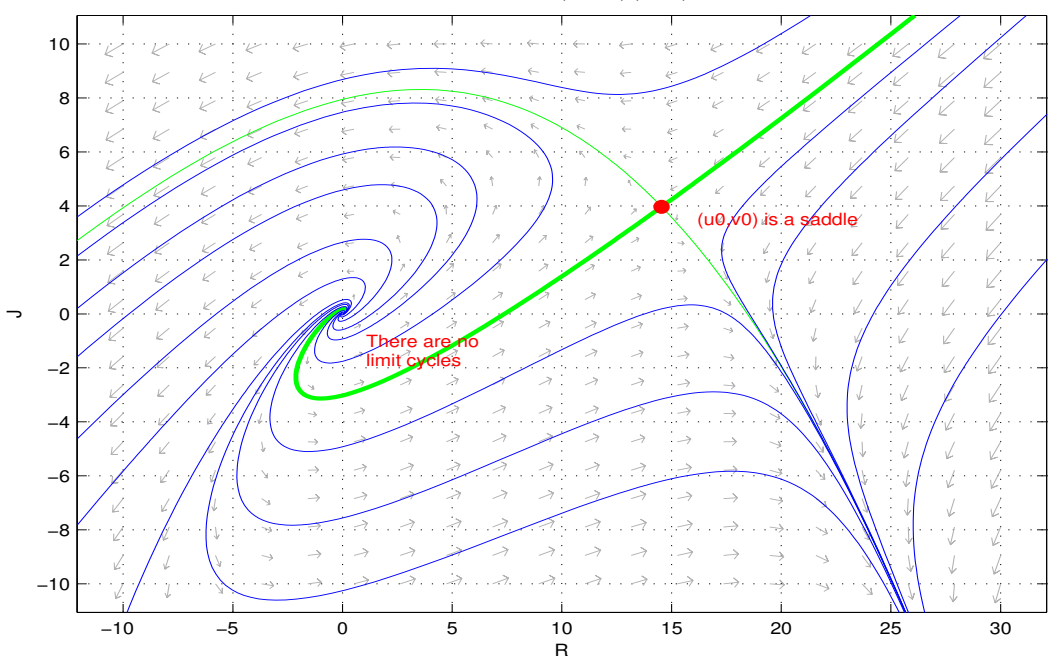

FIGURE 7. The phase plane of system (3.2) in the third case, where $a_{0}=1, b_{0}=1, c_{0}=0.6, d_{0}=0.2, E=20, R_{0}=18$.

system is rich and interesting, see Figure 1 to Figure 4. Also, for the nonlinear love model between two individuals, we introduce the nonlinearities in a different way and investigate the mathematics properties of the system's trajectories and phase-plane, as showing in Theorem 1 and Theorem 2. We also explain its special meanings in a romantic relationship.

\section{Acknowledgments}

The authors express sincere thanks to the editors and anonymous reviewers for their constructive comments and suggestions that helped to improve this paper. This work was supported by the National Natural Science Foundation of China (Grant No. 11301277), the Natural Science Foundation of Jiangsu Province (Grant No. BK20151523), the Six Talent Peaks Project in Jiangsu Province (Grant No. 2015XCL-020), and the Qing Lan Project of Jiangsu Province.

\section{APPENDIX}

Proof of Theorem 1

Define Dulac function as $B(u, v)=\exp (k u+l v)$ and let

$$
k \frac{a_{0}}{b_{0}}+\frac{c_{0}}{b_{0}} l-\frac{2 a_{0}}{b_{0}}=k+\frac{d_{0}}{b_{0}} l=0,
$$


then

$$
\begin{aligned}
D & =\frac{\partial\left[\frac{a_{0}}{b_{0}} u(1-u)-v\right] \exp (k u+l v)}{\partial u}+\frac{\partial\left[-\frac{d_{0}}{b_{0}} v+\frac{c_{0} E}{R_{0} b_{0}}\left(\frac{R_{0}}{E}-u\right) u\right] \exp (k u+l v)}{\partial v} \\
& =\left(\frac{a_{0}-d_{0}}{b_{0}}-\left(k \frac{a_{0}}{b_{0}}+\frac{c_{0} E}{b_{0} R_{0}} l\right) u^{2}\right) \exp (k u+l v) .
\end{aligned}
$$

So when $a_{0} \leq d_{0}$, we may easy get $D \leq 0$, and the equality holds up if and only if $a_{0}=d_{0}$ and $u=0$. It follows from Dulac's principle that system (3.3) has no closed path curve.

Then, when we set

$$
\begin{aligned}
& \frac{a_{0}}{b_{0}} u(1-u)-v=0, \\
& -\frac{d_{0}}{b_{0}} v+\frac{c_{0} E}{b_{0} R_{0}}\left(\frac{R_{0}}{E}-u\right) u=0 .
\end{aligned}
$$

We can obtain the finite singular points $(0,0)$ and

$$
\left(u_{0}, v_{0}\right)=\left(\frac{\left(a_{0} d_{0}-b_{0} c_{0}\right) R_{0}}{a_{0} d_{0} R_{0}-b_{0} c_{0} E}\right.
$$

$\left.\frac{\left(a_{0}-b_{0}\right)\left(a_{0} d_{0}-b_{0} c_{0}\right) R_{0}}{b_{0}\left(a_{0} d_{0} R_{0}-b_{0} c_{0} E\right)}\right)$. By calculating, we know that $\left(u_{0}, v_{0}\right)$ is in the first quadrant.

Moreover, let $\xi=u-u_{0}, \eta=v-v_{0}$, and replaces $\tau$ with $t$, then system (3.3) can be rewritten as

$$
\begin{aligned}
& \frac{\mathrm{d} \xi}{\mathrm{d} t}=\frac{a_{0}}{b_{0}}\left(1-2 u_{0}\right) \xi-\eta-\frac{a_{0}}{b_{0}} \xi^{2}, \\
& \frac{\mathrm{d} \eta}{\mathrm{d} t}=\frac{c_{0} E}{b_{0} R_{0}}\left(\frac{R_{0}}{E}-2 u_{0}\right) \xi-\frac{d_{0}}{b_{0}} \eta-\frac{c_{0} E}{b_{0} R_{0}} \xi^{2} .
\end{aligned}
$$

The characteristic equation of the linear approximate system of (4.3) is

$$
\lambda^{2}+\left[\frac{d_{0}}{b_{0}}-\frac{a_{0}}{b_{0}}\left(1-2 u_{0}\right)\right] \lambda+\frac{c_{0} E}{b_{0} R_{0}}\left(\frac{R_{0}}{E}-2 u_{0}\right)-\frac{a_{0} d_{0}}{b_{0}^{2}}\left(1-2 u_{0}\right)=0 .
$$

By calculating, we know the eigenvalues $\lambda_{1}$ and $\lambda_{2}$ are real and with contrary sign, so $\left(u_{0}, v_{0}\right)$ is a saddle.

For the point $(0,0)$, the characteristic equation is

$$
\lambda^{2}+\frac{d_{0}-a_{0}}{b_{0}} \lambda+\frac{c_{0} b_{0}-a_{0} d_{0}}{b_{0}^{2}}=0 .
$$

We can obtain that the eigenvalues $\lambda_{1}$ and $\lambda_{2}$ are two conjugate complex number with the real part $\frac{a_{0}-d_{0}}{2 b_{0}}<0$, thus $(0,0)$ is a stable strong focus when $a_{0}<d_{0}$. 


\section{Proof of Theorem 2}

By nonsingular transformation, and replaces $\tau$ with $t$, system (3.3) can be rewritten as

$$
\begin{aligned}
& \frac{\mathrm{d} x}{\mathrm{~d} t}=M x-\eta+L x^{2}, \\
& \frac{\mathrm{d} \eta}{\mathrm{d} t}=x(1+A x),
\end{aligned}
$$

where

$$
M=\frac{a_{0}-d_{0}}{\sqrt{c_{0} b_{0}-a_{0} d_{0}}}, L=\frac{a_{0}}{\sqrt{c_{0} b_{0}-a_{0} d_{0}}}, A=\frac{c_{0} b_{0} E-a_{0} d_{0} R_{0}}{\left(c_{0} b_{0}-a_{0} d_{0}\right) R_{0}} .
$$

We know that the phase plane of system (4.6) and system (3.3) are topological homeomorphism. The characteristic equation of the linear approximation system of (4.6) is $\lambda^{2}-M \lambda+1=0$, thus when $a_{0}>d_{0}$, the eigenvalues are two conjugate complex number with the real part $\frac{M}{2}>0$, so $(0,0)$ is an unstable strong focus.

When $a=b, M=0$, system (4.6) is a special case of the following system

$$
\begin{aligned}
& \frac{\mathrm{d} x}{\mathrm{~d} t}=-y+a_{20} x^{2}+a_{11} x y+a_{02} y^{2}, \\
& \frac{\mathrm{d} y}{\mathrm{~d} t}=x+b_{20} x^{2}+b_{11} x y+b_{02} y^{2} .
\end{aligned}
$$

It is easy to compute the first quantities of focus

$$
W_{1}=\frac{1}{3}\left(a_{20}+a_{02}\right)\left(a_{11}+2 b_{02}\right)-\left(b_{20}+b_{02}\right)\left(b_{11}+2 a_{20}\right)=-\frac{2}{3} A L<0 .
$$

Thus, $(0,0)$ is a stable weak focus.

According to Hopf bifurcation theory, when $0<a_{0}-d_{0} \ll b_{0}$, there exists at least one stable limit cycle around $(0,0)$. Also, system (4.6) is the special case of the second class of the second-order differential system studied by Ye [21] as

$$
\begin{aligned}
& \frac{\mathrm{d} x}{\mathrm{~d} t}=-y+M x+L x^{2}+m x y+n y^{2}, \\
& \frac{\mathrm{d} y}{\mathrm{~d} t}=x(1+A x)(A \neq 0) .
\end{aligned}
$$

Thus when $M \leq 0$ or $M \geq \frac{L}{A}$, system (4.6) (and then system (3.3)) has no limit cycles; when $M \in\left(0, \frac{L}{A}\right)$, system (3.3) at most has only one limit cycle and if the limit cycle exist, it must be the single stable cycle around origin point $(0,0)$. So, when $0<a_{0}-d_{0} \ll b_{0}$, system (3.3) exists the only single stable limit cycle around the point $(0,0)$. When $M \geq \frac{L}{A}$, i.e., $a_{0}-d_{0}>\frac{a_{0}\left(a_{0} d_{0}-b_{0} c_{0}\right) R_{0}}{b_{0}\left(a_{0} d_{0} R_{0}-b_{0} c_{0} E\right)}$, system (3.3) has no limit cycles. 


\section{REFERENCES}

[1] F. Bagarello and F. Oliveri, "An operator-like description of love affairs," SIAM J. Appl. Math., vol. 70, no. 8, pp. 3235-3251, 2010, doi: 10.1137/10079985X.

[2] K. Barley and A. Cherif, "Stochastic nonlinear dynamics of interpersonal and romantic relationships," Appl. Math. Comput., vol. 217, no. 13, pp. 6273-6281, 2011, doi: 10.1016/j.amc.2010.12.117.

[3] M. L. Barnes and R. J. Sternberg, The psychological of love. New Haven, C. T. Yele University Press, 1988.

[4] N. Bielczyk, M. Bodnar, and U. Foryś, "Delay can stabilize: love affairs dynamics," Appl. Math. Comput., vol. 219, no. 8, pp. 3923-3937, 2012, doi: 10.1016/j.amc.2012.10.028.

[5] J. M. Gottman, J. D. Murray, C. C. Swanson, R. Tyson, and K. R. Swanson, The mathematics of marriage: dynamic nonlinear models, ser. A Bradford Book. MIT Press, Cambridge, MA, 2002.

[6] A. Gragnani, S. Rinaldi, and G. Feichtinger, "Cyflic dynamics in romantic relationships," International Journal of Bifurcation and Chaos, no. 7, pp. 2611-2619, 1997, doi: 10.1142/S0218127497001771.

[7] W. Liu and K. Chen, "Chaotic behavior in a new fractional-order love triangle system with competition,” J. Appl. Anal. Comput., vol. 5, no. 1, pp. 103-113, 2015.

[8] M. J. Radzicki, "Dyadic processes, tempestuous relationships and system dynamics," System Dynamics Review, no. 9, pp. 79-94, 1993, doi: 10.1002/sdr.4260090105.

[9] A. Rapoport, Fights, games and debates, Ann. Arbor. University of Michigan Press, 1960.

[10] S. Rinaldi, "Love dynamics: the case of linear couples," Appl. Math. Comput., vol. 95, no. 2-3, pp. 181-192, 1998, doi: 10.1016/S0096-3003(97)10081-9.

[11] S. Rinaldi and A. Gragnani, "Love dynamics between secure individuals: A modeling approach," Nonlinear Dynamics, Psychology, and Life Sciences, vol. 2, no. 4, pp. 283-301, 1998, doi: 10.1023/A:1022935005126.

[12] W.-S. Son and Y.-J. Park, "Delayed feedback on the dynamical model of a financial system," Chaos Solitons Fractals, vol. 44, no. 4-5, pp. 208-217, 2011, doi: 10.1016/j.chaos.2011.01.010.

[13] W.-S. Son and Y.-J. Park, "Time delay effect on the love dynamical model," Journal of the Korean Physical Society, vol. 59, no. 3, pp. 2197-2204, 2011.

[14] J. C. Sprott, "Dynamical models of love," Nonlinear Dynamics, Psychology, and Life Sciences, vol. 8, pp. 303-314, 2004.

[15] R. J. Sternberg, “The triangular theory of love,” Psychological Review, vol. 93, pp. 119-135, 1986, doi: 10.1037/0033-295X.93.2.119.

[16] S. H. Strogatx, Nonlinear dynamics and chaos: with applications to physics, biology, chemistry and engineering. Addison- Wesley, Reading, MA, 1994.

[17] J. Sunday, D. J. Zirra, and M. Mijinyawa, "A computational approach to dynamical love model: The Romeo and Juliet scenario," Int. J. Pure Appl. Sci. Technol., vol. 11, no. 2, pp. 10-15, 2012.

[18] S. H. Wang, "Mathematical modeling for the synthetical national power," Appl. Math. J. Chinese Univ. Ser. A, vol. 12, no. 1, pp. 29-36, 1997.

[19] J. Wauer, D. Schwarzer, G. Q. Cai, and Y. K. Lin, "Dynamical models of love with timevarying fluctuations," Appl. Math. Comput., vol. 188, no. 2, pp. 1535-1548, 2007, doi: 10.1016/j.amc.2006.11.026.

[20] Y. Xu, R. C. Gu, and H. Q. Zhang, "Effects of random noise in a dynamical model of love," Chaos Solitons \& Fractals, vol. 44, pp. 490-497, 2011, doi: http://dx.doi.org/10.1016/j.chaos.2011.03.009.

[21] Y. Q. Ye, Limit cycle theory. Shanghai Science and Technology Press, 1984. 
Authors' addresses

\section{Kewang Chen}

College of Mathematics and Statistics, Nanjing University of Information Science and Technology, Nanjing 210044, China

E-mail address: eager4@163.com

Wenjun Liu

College of Mathematics and Statistics, Nanjing University of Information Science and Technology, Nanjing 210044, China

E-mail address: wjliu@nuist.edu.cn

Jaekeun Park

Department of Mathematics, Hanseo University, Chungnam-do, Seosan-si 356-706, Republic of Korea

E-mail address: jkpark@hanseo.ac.kr 